\title{
ROBUST IMAGE STEGNOGRAPHY USING DWT AND DCT
}

\author{
Vasudha', Chrispin Jiji ${ }^{2}$ \\ ${ }^{I}$ Student, Department of ECE, The Oxford College of Engineering, Karnataka, Bangalore. \\ ${ }^{2}$ Assistant Professor, Department of ECE, The Oxford College of Engineering, Karnataka, Bangalore
}

\begin{abstract}
Most imaportant area in the recent years is the field of stegnography. It is the method of hiding data in the cover image, it can be done through the form of text, video or audio. The information is passed from the sender to the receiver without giving any hint to the external users. LSB technique fail to provide the complete security, in order to overcome this problem DCT is applied. Spatial domain is converted to frequency domain by the use of DCT. Encryption is done uding RSA algorithm. This is a robust method. From the result it will be clear that the proposed method shows the better PSNR value.
\end{abstract}

Keywords: Stegnography, Spatial Domain, Discrete Wavelet Transform, Discrete Cosine Transform, Data Hiding, Robustness, PSNR, MSE.

\section{INTRODUCTION}

The wide use of internet can model the growth in the stenography. In recent years hiding information is the essential process. Hence stegnography plays an important role in the field of internet for security purpose. Stegnography hides the information in the form of image, text, video. The aim of using stegnography is the presence of hidden message is not visible for others. The stegnography includes different techniques such as least significant bit(LSB), discrete cosine transformation(DCT), discrete wavelet transformation(DWT).

There are mainly two types of domain in the stegnography that is spatial and frequency domain. Spatial domain involves the application of procees directly on the pixel whereas the frequency domain is the application of process to the co-efficients which is transformed. LSB technique is used in the spatial domain and DCT,DWT are used in the frequency domain. Each pixel is converted into binary value in the LSB tehchnique but it is sensitive to the external attacks.

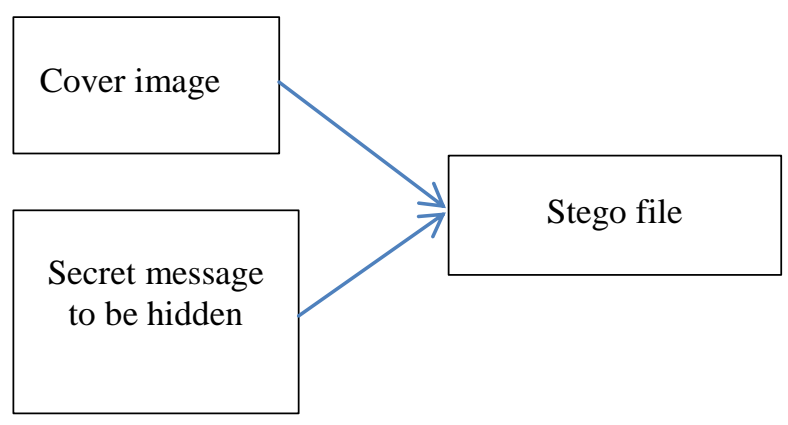

DCT and DWT are the mathematical operations performed to transform the digital image. DCT is used for lossycompression, here message is embedded in the least significant bit. DWT is used to increase the robustness and message to be hidden in the high frequency co-efficients.

\section{LITERATURE SURVEY}

J.R.Krenn[1] proposed LSB technique, it states that the hidden data in LSB cannot be noticed.

K.B.Raja[2] stated that the information is being tranfered from source to destination without knowing. It is the combination of DCT and compression technique.

Vijay kumarsharmaet.al[3] worked on algorithm for 8 or 24 bit to give security against various attacks.

Po yuehchenet.a[4] proposed a method of stegnography which hides message in the frequency domain. It is divided based on the image quality and capacity.

Mehboobet.al[5] proposed anovel technique in art and science of stegnography which uses LSB to hide message in coloue image.

Attalla I. Hashadet.al[6] proposed LSB and DCT. And a new technique emerged using DCT insertion method.

T.Narasimmlouet.al[7] proposed DWT based stegnography which states that PSNR value has better value in proposed method compared to other technique.

Nedaraftariet.al[8] proposed a new method of stegnography which combine wavelet and cosine transform to give better quality. 


\section{STEGNOGRAPHY}

Stegnography method hides the secret message using a secret key. It is an adaptive data hiding technique. Each coefficients holds different secret message. Hiding capacity operates according to different application. The basic model of stegnography is as shown in block diagram.

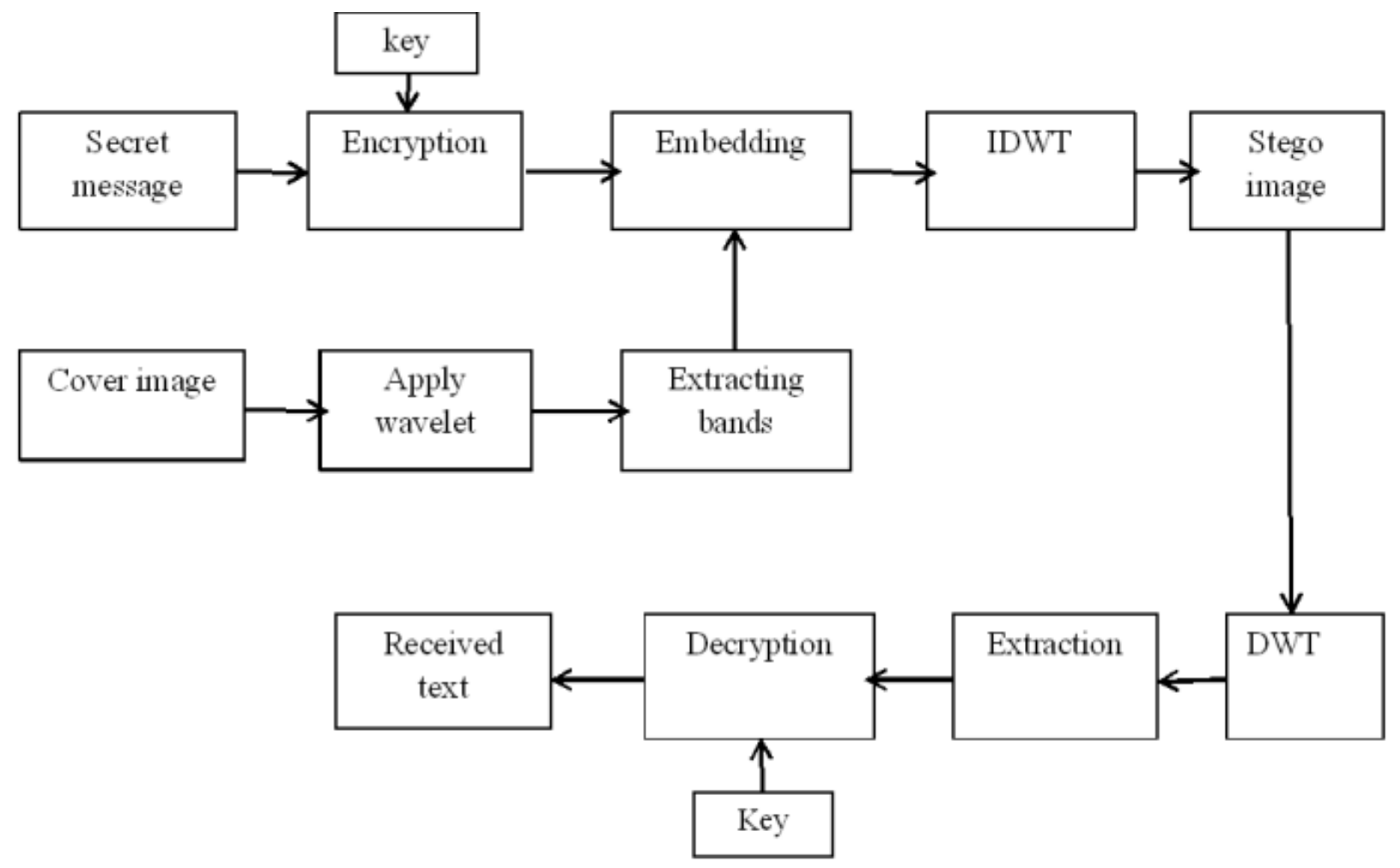

Fig 1: Block diagram

By using stegnographic algorithm secret message is embeeded into the cover image. The result gives the stego image and it is transferred from sender to receiver with particular key. Same method is used for extraction process. Both should agree to the same key usage.

\section{Transform Domain Technique:}

The transform domain techniques make use of the transform coefficients to hide the data. Co-efficients are modified to hide the data. The different techniques used are DCT and DWT. Discrete Cosine Transform (DCT): The discrete cosine transforms (DCT) \& discrete wavelet transform (DWT).

\section{Discrete Wavelet Transform (DWT):}

In DWT, secret messages are embedded in the high frequency coefficients resulted from Discrete Wavelet Transform. It increases the robustness. Haar wavelet has horizontal and vertical operation. It is a tool for image decomposition. Small waves are called wavelets, it is caused due to translation and dilation. 2-D image is classified into four non-overlapping subbands. They are LL.LH.HL,HH.

Here the image is located in the LL subband which has great image regradation. Thus increases the rocustness while embedding in the lower frequency.

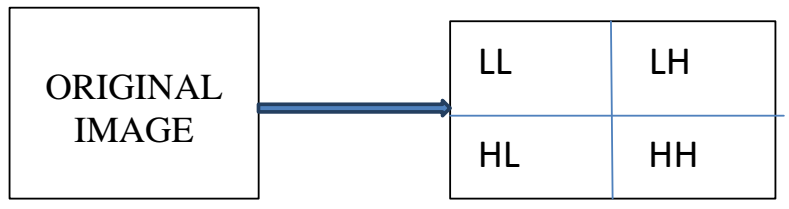

Discrete cosine transformation

DCT is applied for embedding and extraction process on the cover image. It has high compression ration, good integration ability, small bit error rate, and good effect of complexity calculation. It is divided into different frequency bands. It has high,low and mid frequency component. Low frequency has most visual parts of the image. In high frequency components are removed through compression.

\section{PROPOSED SYSTEM}

Both DCT and DWT technique are considered in the proposed method. DWT is used for hidden images and DCT is applied on cover object for embedding and extracting data. Take cover image apply DWT the result is the stego image. Then retrieve the information from the cover image. And then perform DCT operation. Calculate PSNR and MSE value for various images. Proposed method shows the high PSNR value. 


\section{Peak signal to noise ratio:}

It scales the MSE by avoiding the problem of MSE.

$\mathrm{PSNR}=10 \log _{10}(255)^{2} / \mathrm{MSE}$

\section{Mean Square Error:}

$\mathrm{MSE}=\frac{\sum_{M, N}[I 1(m, n)--I 2(m, n)]^{2}}{M * N}$

Where, $\mathrm{M}$ and $\mathrm{N}$ are number of rows and columns in input image

\section{Algorithm}

Step 1: Read the cover image file.

Step 2: Apply histogram modification.

Step 3: Read the input text message that is to be hidden in the cover image.

Step 4: Input text is encrypted using RSA algorithm.

Step 5: Applying wavelet considering LL band for better visuality.

Step 6:Apply discrete cosine transform and SVD

Step 7: Encrypted data is converted into matrix.

Step 8: hide the secrete message in the cover image.

Step 9: Apply inverse discrete cosine transform and ISVD.

Step 10: Stego image is obtained.

\section{Flowchart}

The flowchart shows both embedding and extraction process. Histogram modification is applied in order to prevent overflow/underflow. Transformation is applied for the co-efficients. Inverse is applies for DWT and DCT for the transform domain technique.

At the extraction process same algorithm is used to get the seceret message.

Embedding and Extraction process:
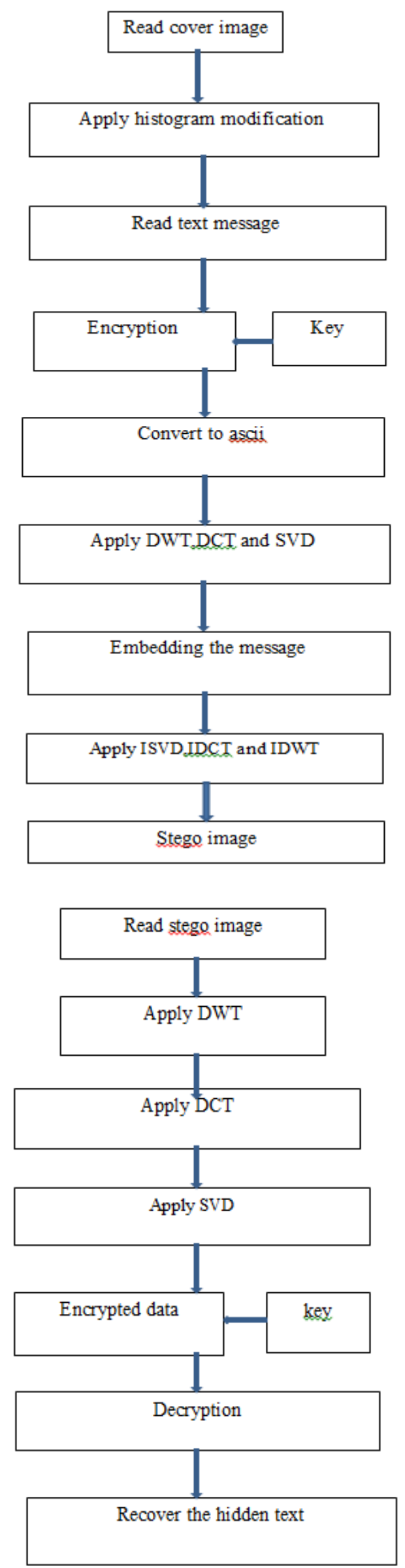


\section{VEXPERIMENTAL RESULTS}

Consider different images, plot histogram and then histogram modification is applied to enhance the brightness. Text is hidden in the cover image and then converted to matrix form by encrypting. Finally the text is received with the hidden message. PSNR, MSE and accuracy is calculated for various images.
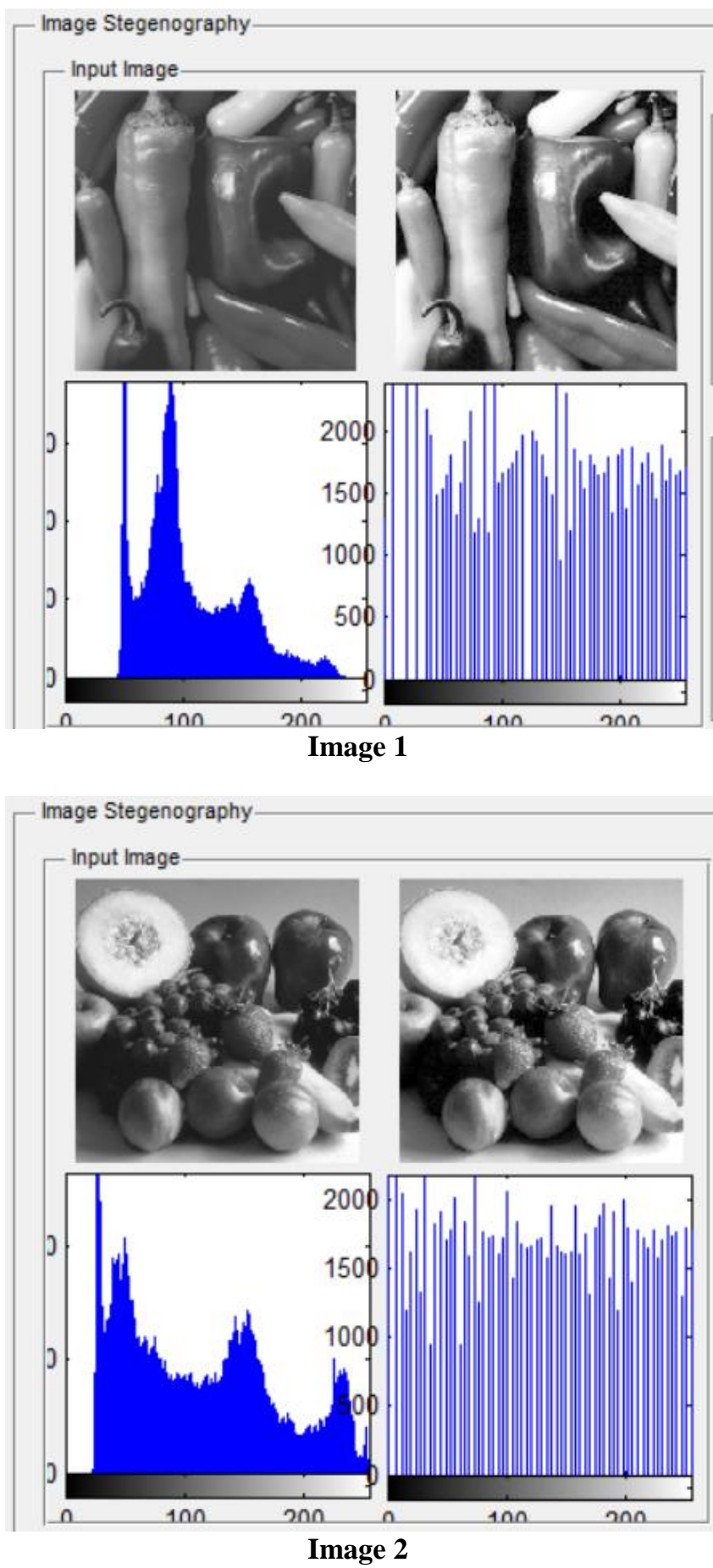

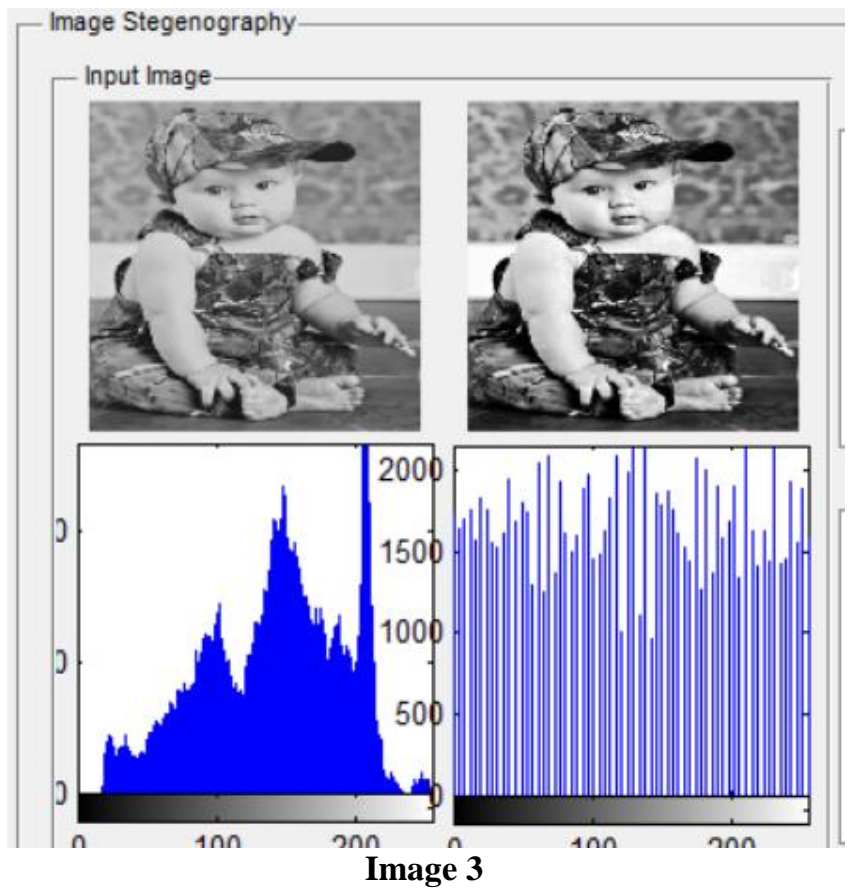

Table1: Existing method

\begin{tabular}{|l|l|l|l|}
\hline & PSNR & MSE & $\mathrm{A}$ \\
\hline Image 1 & 25.899 & 168.466 & 0.83 \\
\hline Image 2 & 25.90 & 168.33 & 0.9 \\
\hline Image 3 & 25.89 & 168.56 & 0.83 \\
\hline
\end{tabular}

Table 2: Proposed method

\begin{tabular}{|l|l|l|l|}
\hline & PSNR & MSE & A \\
\hline $\operatorname{Im} 1$ & 29.78 & 68.83 & 1 \\
\hline $\operatorname{Im} 2$ & 29.80 & 68.54 & 1 \\
\hline $\operatorname{Im} 3$ & 29.77 & 68.97 & 1 \\
\hline
\end{tabular}

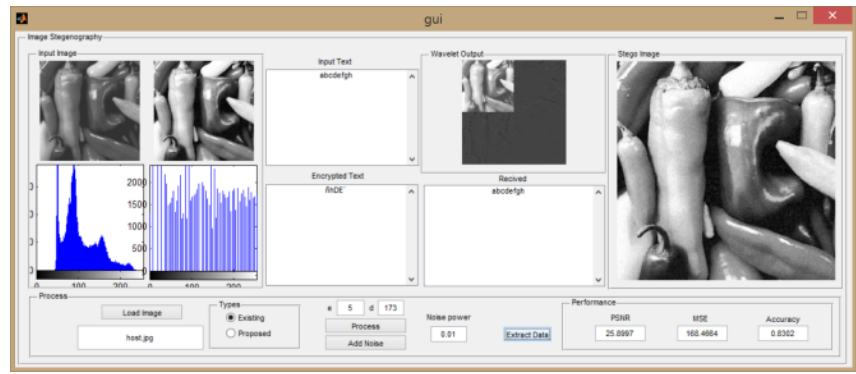

Fig 1: Result of existing method

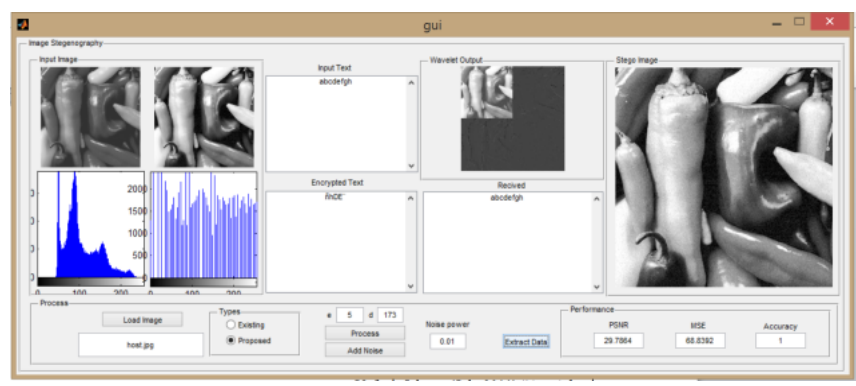

Fig 2: Result of proposed method 


\section{CONCLUSION}

Adaptive data hiding scheme is used in which the hiding capacity is increased. This method embeds more data. The secret key which is known only to sender and receiver. Image quality is increased with the increase in the PSNR value. Hence the robustness is increased. This proves the value of PSNR is more in the proposed system than the other techniques.

\section{REFERENCES}

[1] J.R. Krenn, "Steganography and Steganalysis", January 2004.

[2] K.B.Raja, C.R.Chowdary, Venugopal K R, L.M.Patnaik, "A Secure Image Steganography using LSB, DCT and Compression Techniques on Raw Images", IEEE-0-78039588-3/05/\$20.00 @2005.

[3] Vijay Kumar Sharma, Vishal shrivastava, "A Steganography Algorithm for Hiding Images by improved LSB substitution by minize detection." Journal of Theoretical and Applied Information Technology, Vol. 36 No.1, ISSN: 1992-8645, 15th February 2012.

[4] Po-Yueh Chen and Hung-Ju Lin, "A DWT Based Approach for Image Steganography", International Journal of Applied Science and Engineering 4, 3: 275-290, 2006.

[5] BeenishMehboob and Rashid Aziz Faruqui, "A Steganography Implementation”, IEEE -4244-24276/08/\$20.00 @2008.

[6] Atalla I. Hashad,Ahmed S. Madani, "A Robust Steganography Technique Using Discrete Cosine Transform Insertion"

[7] T. Narasimmalou, Allen Joseph .R, “Optimized Discrete Wavelet Transform based Steganography" , IEEE International Conference on Advanced Communication Control and Computing Technologies (ICACCCT), 2012.

[8] NedaRaftari and Amir MasoudEftekhariMoghadam, "Digital Image Steganography Based on Assignment Algorithm and Combination of DCT-IWT", Fourth International Conference on Computational Intelligence, Communication Systems and Networks, 2012.

\section{BIOGRAPHIES}

Vasudha.S completed BE in sjmitchitradurga in electronics and communication department. Mtech in oxford college of engineering, Bangalore.

Chrispinjiji, Asst. Prof, The oxford college of engineering, Bangalore. 